\title{
Exact 2-Distance b-Coloring and Exact 2-Distance b-Continuity of Helm Graph $\boldsymbol{H}_{n}$
}

\author{
S. Saraswathi ${ }^{1}$, M. Poobalaranjani ${ }^{2}$ \\ ${ }^{1,2} P G$ \& Research Department of Mathematics \\ Seethalakshmi Ramaswami College (Autonomous) \\ Affiliated to Bharathidasan University, Tiruchirappalli, Tamil Nadu, India.
}

\begin{abstract}
An exact 2-distance coloring of a graph $G$ is a coloring of vertices of $G$ such that any two vertices which are at distance exactly 2 receive distinct colors. An exact 2-distance chromatic number $\chi_{e 2}(G)$ of $G$ is the minimum $k$ for which $G$ admits an exact 2-distance coloring with $k$ colors. A b-coloring of $G$ by $k$ colors is a proper $k$-vertex coloring such that in each color class, there exists a vertex called a color dominating vertex which has a neighbor in every other color class. A vertex that has a 2-neighbor in all other color classes is called an exact 2-distance color dominating vertex (or an e2-cdv). Exact 2-distance b-coloring (or an $e 2 b$-coloring) of $G$ is an exact 2-distance coloring such that each color class contains an e2$c d v$. An exact 2-distance b-chromatic number (or an e2b-number) $\chi_{e 2 b}(G)$ of $G$ is the largest integer $k$ such that $G$ has an e $2 b$-coloring with kcolors. If for each integerk, $\chi_{e 2}(G) \leq k \leq$ $\chi_{e 2 b}(G), G$ has an e2b-coloring by $k$ colors, then $G$ is said to be an exact 2-distance $b$ continuous graph. In this paper, the e2b-number $\chi_{e 2 b}\left(H_{n}\right)$ of the helm graph $H_{n}$ is obtained and e2b-continuity of $H_{n}$ is discussed.
\end{abstract}

Keywords: Exact 2-distance b-coloring, exact 2-distance b-chromatic number, exact 2-distance color dominating vertex, exact 2-distance b-continuity.

\section{Introduction}

All graphs considered in this paper are finite, simple and undirected. For those terminologies not defined in this paper, the reader may refer to [1]. In this paper, exact 2distance $b$-coloring of a helm graph is studied. As the name indicates, this coloring is a combination of two types of coloring, viz., exact 2-distance coloring and $b$-coloring. An exact 2-distance coloring is a distance coloring which need not be proper and $b$-coloring is a proper coloring. Distance coloring was introduced by F. Kramer and H. Kramer [4], [5] in 1969. As the name suggests it is based on distance between two vertices. The definition of distance coloring requires the definition of 2-neighbors. If $u$ and $v$ are vertices in $G$ such that $d(u, v)=$ 2 , then $u$ is said to be a 2-neighbor of $v$ and vice versa. The set of all 2 neighbors of $u$ is denoted by $N_{2}(u)$ and is called open 2-neighborhood of $u$ and $N_{2}[u]=N_{2}(u) \cup\{u\}$ is called the closed 2-neighborhood of $u$. The $d_{2}$-degree of a vertex $u$ denotes the number of 2 neighbors of $u$ and $\Delta_{2}(G)$ denotes the maximum $d_{2}$-degree. 
A 2-distance coloring of $G$ is an assignment of colors to vertices such that every pair of vertices $u$ and $v$ such that $d(u, v) \leq 2$ receive distinct colors. The 2-distance chromatic number of $G$, denoted $\chi_{2}(G)$ is the smallest integer $k$ for which $G$ admits a 2-distance $k$ coloring. An exact 2-distance coloring (or $e 2$-coloring) is a distance coloring such that, every pair of vertices $u$ and $v$ such that $d(u, v)=2$ receive distinct colors. Clearly, it need not be a proper coloring and every 2-distance coloring is an $e 2$-coloring. The exact 2-distance chromatic number $\chi_{e 2}(G)$ (or $e 2$-number) of $G$, is the smallest integer $k$ for which $G$ admits an $e 2$-coloring with $k$ colors. The other coloring of interest is $b$-coloring. The concept of $b$ coloring was introduced by Irving and Manlove [2] in 1991. A $b$-coloring of $G$ by $k$-colors is a proper $k$-coloring such that in each color class, there exists a vertex called a color dominating vertex, which has a neighbor in every other color class. The $b$-chromatic number $\chi_{b}(G)$ of $G$ is the largest integer $k$ such that $G$ has a $b$-coloring by $k$ colors. Another interesting idea in $b$-coloring is $b$-continuity. Since, $\chi$-coloring is a $b$-coloring and a $\chi_{b^{-}}$ coloring tries to find the maximum number of colors a $b$-coloring can have, there can be intermediate values between $\chi(G)$ and $\chi_{b}(G)$. If for each intermediate values, there exists a $b$-coloring, then the graph $G$ is said to be $b$-continuous.

We [7] in 2019, made a study on combining these two coloring which resulted as a new coloring called exact 2-distance $b$-coloring. In that paper, the $e 2 b$-number of path, cycle and wheel were obtained. In this paper, the $e 2 b$-number of a helm graph $H_{n}$ is studied, where a helm $H_{n}$ is obtained from an $(n-1)$-cycle such that all the $n-1$ vertices of the cycle are adjacent to a central vertex say $u$, and each vertex on the cycle is adjacent to one pendant vertex. Analogous to $b$-continuity, we defined exact 2 -distance $b$-continuity ( $e 2 b$-continuity) of graphs. When a new concept is introduced, there may be the case that, all graphs may fall into that category. Hence, it is important to prove that, the definition is not trivial. In this paper, we have proved that $H_{4}$ is not $e 2 b$-continuous and $H_{n}, n \geq 5$ is $e 2 b$-continuous.

\section{Definitions and some prior results}

In this section, the definition of the new coloring and the corresponding parameter are stated.

Definition 2.1 [7]. A vertex which has a 2-neighbor in all other color classes is called an exact 2-distance color dominating vertex (or an $e 2-c d v$ ). If $u$ is an $e 2-c d v$ of color $i$, then it is called an $i-e 2-c d v$.

Definition 2.2 [7]. An exact 2-distance $b$-coloring (or an $e 2 b$-coloring) of a graph $G$ is an exact 2-distance coloring ( $e 2$-coloring) of $G$ such that each color class contains an $e 2-c d v$.

An exact 2-distance $b$-chromatic number (or an $e 2 b$-number) $\chi_{e 2 b}(G)$ of $G$ is the largest integer $k$ such that $G$ has an $e 2 b$-coloring with $k$ colors.

i. e. , $\chi_{e 2 b}(G)=\max \{k: G$ has an $e 2 b$-coloring with $k$ colors $\}$. 
Definition 2.3 [7]. The term $m_{2}$-degree denoted by $m_{2}(G)$ of $G$ is defined as the largest integer $k$ such that $G$ has at least $k$ vertices having at least $(k-1)$ 2-neighbors.

i.e.,$m_{2}(G)=\max \left\{k: G\right.$ has at least $k$ vertices whose $d_{2}$-degree is at least $\left.k-1\right\}$.

\section{Observation 2.4 [7].}

(i) $e 2 b$-coloring exists for all graphs;

(ii) For a graph $G, \chi_{e 2}(G) \leq \chi_{e 2 b}(G) \leq m_{2}(G) \leq \Delta_{2}(G)+1$;

Proposition 2.5 [7]. Any minimum $e 2$-coloring of a graph is an $e 2 b$-coloring.

Since, the $e 2 b$-number is the maximum number of colors an $e 2 b$-coloring can have, to find the maximum, minimum number is required. From proposition $2.5, \chi_{e 2}$ is the minimum number required to have an $e 2 b$-coloring. Hence, the $e 2$-number of $H_{n}$ is given in the next proposition.

Proposition 2.6. For $n \geq 4$

$$
\chi_{e 2}\left(H_{n}\right)=\left\{\begin{array}{lc}
2, & \text { if } n=4 \\
3, & \text { if } n=5 \\
4, & \text { if } n=6,7 \\
\left\lfloor\frac{n}{2}\right\rfloor, & \text { if } n \geq 8
\end{array}\right.
$$

\section{Exact 2-distance $\boldsymbol{b}$-coloring of $\boldsymbol{H}_{\boldsymbol{n}}$}

In this section, the bound $\chi_{e 2 b}\left(H_{n}\right)$ is obtained.

Theorem 3.1. For $n \geq 4$,

$$
\chi_{e 2 b}\left(H_{n}\right)= \begin{cases}4, & \text { if } n=4 \\ n-2, & \text { if } n \geq 5\end{cases}
$$

Proof: The $d_{2}$-degree of the vertices of $H_{n}$ are given by

$$
d_{2}(u)=n-1, d_{2}\left(v_{i}\right)=n-2 \text { and } d_{2}\left(w_{i}\right)=3
$$

Claim 1: $m_{2}\left(H_{n}\right)=\left\{\begin{array}{lll}4, & \text { if } n=4 \\ n-1, & \text { if } n \geq 5\end{array}\right.$

Let $n=4$. Then from (1), $u$ and $w_{i}$ constitute a set of $n$ vertices of $d_{2}$-degree $=n-1=3$. Hence, $m_{2}\left(H_{n}\right)=n=4$.

If $n=5$, then both $v_{i}$ and $w_{i}$ are of $d_{2}$-degree $n-2$. Hence, $m_{2}\left(H_{n}\right)=n-1$.

Suppose $n \geq 6$. Then $w_{i}$ does not contribute to $m_{2}\left(H_{n}\right)$. In this case, $u$ and $v_{i}$ constitute a set of $n$ vertices of $d_{2}$-degree at least $n-2$. Hence, $m_{2}\left(H_{n}\right)=n-1$. Thus, summarizing the $m_{2^{-}}$values, the claim is proved.

Throughout the proof, $c: V \rightarrow\{1,2, \ldots, m\}$ is a coloring of $H_{n}$ where $m \geq \chi_{e 2}\left(H_{n}\right)$ and $c(u)=$ 1.

Suppose $n=4$. Then from claim $1, m_{2}\left(H_{n}\right)=4$. Let $c\left(v_{i}\right)=c\left(w_{i}\right)=i, 2 \leq i \leq 4$. Then each $w_{i}$ is an $i-e 2-c d v$ (Refer figure 1). Hence, $\chi_{e 2 b}\left(H_{4}\right)=4$.

Now let $n \geq 5$. The proof is done in 2 stages. 
(a) Proving $H_{n}$ cannot have an $e 2 b$-coloring with $n-1$ colors;

(b) Proving $H_{n}$ has an $e 2 b$-coloring with $n-2$ colors.

Let $D$ denote the set of all $e 2$-color dominating vertices of $H_{n}$.

Claim 2 : For $n \geq 5, \chi_{e 2 b}\left(H_{n}\right) \leq n-2$. i.e, $H_{n}$ cannot have an $e 2 b$-coloring with $n-1$ colors

Suppose $c$ is an $e 2 b$-coloring of $H_{n}$ with $n-1$ colors. We discuss 2 cases.

\section{Case (i): $n=5$}

Then $c$ is an $e 2 b$-coloring of $H_{n}$ with 4 colors and $d_{2}(u)=4 \& d_{2}\left(v_{i}\right)=d_{2}\left(w_{i}\right)=3$. Hence, each vertex of $V$ can be an $e 2-c d v$. Then if $v \in D, v \neq u$, then $N_{2}(v)$ receives distinct colors. Since $c(u)=1, c\left(w_{i}\right) \neq 1$ for any $i$. Hence, $D$ cannot be a set of $w_{i}{ }^{\prime} s$.

Sub claim 1: If $w_{i} \in D$, then $w_{i+2} \in D$ and $c\left(w_{i}\right)=c\left(w_{i+2}\right)$

Since $w_{i} \in D, N_{2}\left[w_{i}\right]$ receives 4 distinct colors. Hence, as $N_{2}\left(w_{i+2}\right)=N_{2}\left(w_{i}\right)=$ $\left\{u, v_{i-1}, v_{i+1}\right\}, N_{2}\left(w_{i+2}\right)$ receives 3 distinct colors. Since $c$ is an $e 2$-coloring, $c\left(w_{i+2}\right) \notin$ $C\left(N_{2}\left(w_{i+2}\right)\right)$ and as $c$ is a 4-coloring, $c\left(w_{i+2}\right) \in\{1,2,3,4\}-C\left(N_{2}\left(w_{i+2}\right)\right)=\{1,2,3,4\}-$ $C\left(N_{2}\left(w_{i}\right)\right)=\left\{c\left(w_{i}\right)\right\}$. Hence, $c\left(w_{i+2}\right)=c\left(w_{i}\right)$ and $N_{2}\left[w_{i+2}\right]$ receives 4 distinct colors. This gives $w_{i+2} \in D$.

Sub claim 2: If $v_{i} \in D$ of color $r \neq 1$, then the following hold.

(i) If $v \neq v_{i}$ and $N_{2}\left[v_{i}\right]=N_{2}[v]$, then $v \in D$;

(ii) $c\left(v_{i+2}\right)=1$ and $v_{i+2}$ is a $1-e 2-\mathrm{cdv}$;

(iii) $w_{i-1}$ and $w_{i+1}$ cannot be $e 2$-color dominating vertices;

(iv) If $c\left(v_{j}\right) \neq 1$ and $j \neq i$, then $v_{j} \notin D$.

Proof of (i): Since $v_{i} \in D, C\left(N_{2}\left[v_{i}\right]\right)=\{1,2,3,4\}$. If $N_{2}\left[v_{i}\right]=N_{2}[v]$, then $C\left(N_{2}[v]\right)=$ $\{1,2,3,4\}$. Hence, $v$ is an e2-cdv.

Proof of (ii): Since $v_{i} \in D, v_{i}$ has a 2-neighbor in each color class and hence in $S_{1}$.

Since $u \in S_{1}$ is not a 2-neighbor of $v_{i}$ and $w_{j} \notin S_{1}$ for any $j, c\left(v_{j}\right)=1$ for some $j$. Since, $v_{i+2}=v_{i-2}$ is the only 2-neighbor of $v_{i}$ among $v_{j}^{\prime} s, c\left(v_{i+2}\right)=1$. As $N_{2}\left[v_{i+2}\right]=$ $\left\{v_{i}, v_{i+2}, w_{i-1}, w_{i+1}\right\}=N_{2}\left[v_{i}\right]$, by (i), $v_{i+2} \in D$.

Proof of (iii): Now $N_{2}\left(w_{i-1}\right)=N_{2}\left(w_{i+1}\right)=\left\{u, v_{i}, v_{i+2}\right\}$ and $c(u)=c\left(v_{i+2}\right)=1$ implies that the 2-neighbors of both $w_{i-1}$ and $w_{i+1}$ are not receiving distinct colors. Hence, they cannot be $e$-color dominating vertices.

Proof of (iv): Suppose $v_{j} \in D$ for $j \neq i$ and $c\left(v_{j}\right) \neq 1$. Since $j \neq i, v_{j} \neq v_{i}$ and as $c\left(v_{j}\right) \neq 1$, from (ii), $v_{j} \neq v_{i+2}$. Therefore, either $v_{j}=v_{i-1}$ or $v_{j}=v_{i+1}$. Without loss of generality, let $v_{j}=v_{i-1}$. Then by (ii), $v_{j+2}=v_{i+1}$ is a 1-e2-cdv. From (iii), $w_{i-2}=w_{i+2}$ and $w_{i}$ cannot be $e 2$-color dominating vertices. Hence, none of the $w_{i}$ is an $e 2-\mathrm{cdv}$ and hence they cannot be members of $D$. Since $v_{i} \& v_{i-1}$ are the only two $e 2$ color dominating vertices of color other than $1, D$ contains $e 2$ color dominating vertices for at most 3 colors, a contradiction to $c$ is a 4-e $2 b$-coloring. Thus, (iv) holds and hence the proof of sub claim 1 is complete.

From sub claim 1 , it is clear than $D$ contains only even number of $w_{i}{ }^{\prime} s$. If $D$ does not contain any $w_{i}$, then $D$ must contain $v_{i}$ 's for at least 3 distinct colors, and hence contain at least three $v_{i}$ 's, a contradiction to (iv) of sub claim 2. Suppose $D$ contains two $w_{i}$ 's. Then from sub claim 1 , they are of same color. Then $D$ must contain $v_{i}{ }^{\prime} s$ for at least 2 distinct colors, and hence 
contain at least two $v_{i}{ }^{\prime} s$, again a contradiction to (iv) of sub claim 2. Suppose, four $w_{i}$ 's. Then from sub claim 1 , they are of 2 distinct colors. Then $D$ must contain at least one $v_{i}$ for a color other than 1 . If $v_{i} \in D$, then by (ii) of sub claim $2, w_{i-1}, w_{i+1} \notin D$, a contradiction to the choice of $D$. Hence, $H_{n}$ cannot have an $e 2 b$-coloring with $n-1=4$ colors.

Case (ii): $n \geq 6$

Then $m_{2}\left(H_{n}\right)=\chi_{e 2 b}\left(H_{n}\right)=n-1 \geq 5$ and as $d_{2}\left(w_{i}\right)=3, w_{i}$ cannot be an $e 2-c d v$, for any $i$. Since, $c$ is an $(n-1)$-coloring and $c(u)=1$, at least $(n-2) v_{i}{ }^{\prime} s$ are $e 2$-color dominating vertices of distinct colors other than 1 . Hence, at most one $v_{i}$ can be of color 1 . As an $r$ - $e 2-$ cdv, $r \neq 1$ requires a 2 -neighbor of color 1 , as in case (i), at least a $v_{j}$ must be of color 1 . Since $(n-2) v_{i}$ 's are of distinct colors other than 1 , only one $v_{j}$ can be of color 1 . Without loss of generality, let $c\left(v_{j}\right)=1$. Then $v_{j-1}$ and $v_{j+1}$ cannot have a 2 -neighbor of color 1 , a contradiction.

Hence from subcases (i) and (ii), $H_{n}$ cannot have an $e 2 b$-coloring with $n-1$ colors and hence, claim 2 is completed.

Hence, the first stage (a) of the proof that, proving $H_{n}$ cannot have an $e 2 b$-coloring with $n-1$ colors is completed.

Now let us prove (b) that $H_{n}$ has an $e 2 b$-coloring with $n-2$ colors.

Case (i): $n=5,6$

From proposition 2.6, $\chi_{e 2}\left(H_{n}\right)=n-2$. Then by claim $2, n-2 \leq \chi_{e 2 b}\left(H_{n}\right) \leq n-2$ and the equality holds.

Case (ii): $n=7$

Then by the same propositions of case (i), $\chi_{e 2}\left(H_{n}\right)=4$ and by claim $2, \chi_{e 2 b}\left(H_{n}\right) \leq n-2=$ $5,4 \leq \chi_{e 2 b}\left(H_{n}\right) \leq 5$. From figure $2, H_{7}$ has an $e 2 b$-coloring with 5 colors and hence, $\chi_{e 2 b}\left(H_{7}\right)=5$.

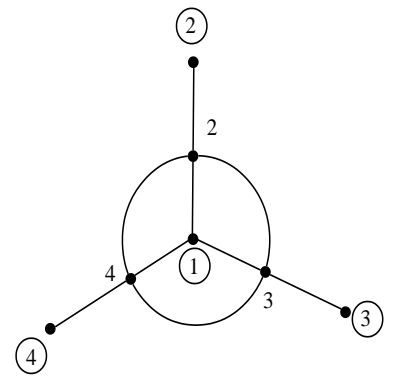

Figure 1: A 4- $e 2 b$-coloring of $\mathrm{H}_{4}$

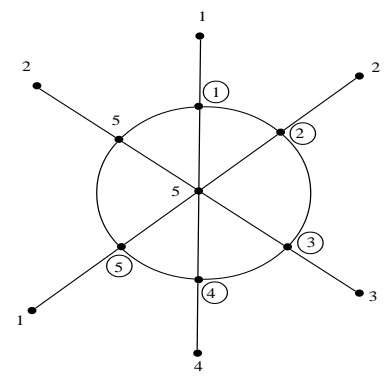

Figure 2: A 5-e2bcoloring of $\mathrm{H}_{7}$

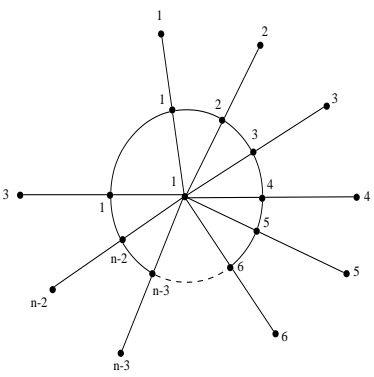

Figure 3: An $(n-2)$-coloring of $H_{n}$

\section{Case (iii): $n \geq 8$}

Let $c$ be defined with $(n-2)$-colors as in (2).

$$
\left.\begin{array}{l}
c(u)=1 \\
c\left(v_{i}\right)=c\left(w_{i}\right)=i, \quad 2 \leq i \leq n-2 \\
c\left(v_{1}\right)=c\left(v_{n-1}\right)=1 \\
c\left(w_{1}\right)=c\left(w_{n-1}\right)=3
\end{array}\right\}
$$

(Refer figure 4). First, let us verify this is an $e 2$-coloring. On the $(n-1)$-cycle, other than $v_{1}$ and $v_{n-1}$ all are of distinct colors. Hence, on $W_{n} e 2$-coloring holds. Hence, it is enough to 
check for each $i, c\left(v_{i}\right) \neq c\left(w_{i-1}\right), c\left(w_{i+1}\right)$. For $3 \leq i \leq n-3,4 \leq i+1 \leq n-2$ and $2 \leq$ $i-1 \leq n-4$. Hence, $c\left(v_{i}\right)=i, c\left(w_{i-1}\right)=i-1 \& c\left(w_{i+1}\right)=i+1$.

$\therefore$ for $3 \leq i \leq n-3, c\left(v_{i}\right) \neq c\left(w_{i-1}\right), c\left(w_{i+1}\right)$

For $i=1,2,-2, n-1, c\left(v_{i}\right), c\left(w_{i-1}\right) \& c\left(w_{i+1}\right)$ are tabulated in table 1 .

Table 1: Colors of $v_{i}, w_{i-1} \& w_{i+1}$

\begin{tabular}{|c|c|c|c|}
\hline $\boldsymbol{i}$ & $\boldsymbol{c}\left(\boldsymbol{v}_{\boldsymbol{i}}\right)$ & $\boldsymbol{c}\left(\boldsymbol{w}_{\boldsymbol{i}-\mathbf{1}}\right)$ & $\boldsymbol{c}\left(\boldsymbol{w}_{\boldsymbol{i}+\mathbf{1}}\right)$ \\
\hline 1 & 1 & 3 & 2 \\
\hline 2 & 2 & 3 & 3 \\
\hline$n-2$ & $n-2$ & $n-3$ & 3 \\
\hline$n-1$ & 1 & $n-2$ & 3 \\
\hline
\end{tabular}

From table $1, c\left(v_{i}\right) \neq c\left(w_{i-1}\right), c\left(w_{i+1}\right)$ for the specified values of $i$. Hence, from (3) and from table $1, c$ is an $e 2$-coloring. Clearly, $u$ is a $1-e 2-c d v$ and for $i, 2 \leq i \leq n-2, v_{i}$ is an $i$ - $e 2$ cdv. Hence, $H_{n}$ admits an $e 2 b$-coloring with $n-2$ colors and hence, $\chi_{e 2 b}\left(H_{n}\right)=n-2$.

\section{4. $\quad$ Exact 2-distance $b$-continuity of Graphs}

In this section, analogous to the definition of $b$-continuity, we introduce the concept of exact 2 -distance $b$-continuity of graphs. In connection with the concept, some more definitions are in need. Necessary new definitions are defined. As a main result, the exact 2-distance $b$ continuity of a Helm graph $H_{n}$ is discussed.

Definition 4.1. If for each integer $k, \chi_{e 2}(G) \leq k \leq \chi_{e 2 b}(G), G$ has a $k$-e $2 b$-coloring then $G$ is said to be an exact 2-distance $b$-continuous graph (or $e 2 b$-continuous graph) or we say $G$ is $e 2 b$-continuous.

Definition 4.2. The exact 2-distance $b$-spectrum (or $e 2 b$-spectrum) $S_{e 2 b}(G)$ of $G$ is defined by the set of all $k$ such that $G$ has an $e 2 b$-coloring by $k$ colors.

In other words, $S_{e 2 b}(G)=\{k: G$ has an $k-e 2 b$-coloring $\}$.

Thus, if $S_{e 2 b}(G)$ contains all the integers from $\chi_{e 2}(G)$ to $\chi_{e 2 b}(G)$, then $G$ is $e 2 b$-continuous.

\section{Theorem 4.3.}

(i) For $n=4, H_{n}$ is not $e 2 b$-continuous and $S_{e 2}\left(H_{n}\right)=\{2,4\}$.

(ii) For $n \geq 5, H_{n}$ is $e 2 b$-continuous and

$$
S_{e 2 b}\left(H_{n}\right)=\left\{\begin{aligned}
\{3\}, & \text { if } n=5 \\
\{4\}, & \text { if } n=6 \\
\{4,5\}, & \text { if } n=7 \\
\left\{\left\lfloor\frac{n}{2}\right\rfloor,\left\lfloor\frac{n}{2}\right\rfloor+1, \ldots, n-2\right\}, & \text { if } n \geq 8
\end{aligned}\right.
$$

Proof:

(i) From proposition 2.6 and theorem 3.1, $\chi_{e 2}\left(H_{4}\right)=2 \& \chi_{e 2 b}\left(H_{n}\right)=4$. Hence, to check the $e 2 b$-continuity, the existence of an $e 2 b$ - coloring with 3 colors has to be checked.

So suppose $c$ is an $e 2 b$-coloring with 3 colors. From equation $(1), d_{2}(u)=d_{2}\left(w_{i}\right)=3$ and $d_{2}\left(v_{i}\right)=2$. Hence, any $v \in V$ can be an $e 2-c d v$. As always let $c(u)=1$. Since, $N_{2}\left(v_{i}\right)=$ 
$\left\{w_{i-1}, w_{i+1}\right\}$, and $c\left(w_{j}\right) \neq 1$ for each $j$, a $v_{i}$ of color $r \neq 1$, cannot have 2 -neighbor of color 1 . Hence, a $v_{i}$ can neither be a 2-e2-cdv nor a 3-e2-cdv. Hence, a 2- $e 2-c d v$ and a $3-e 2-c d v$ occur among $w_{i}{ }^{\prime} s$ only. Since, $w_{i}$ can be given either color 2 or 3 , and there are only $3 w_{i}$ 's, one color appears twice and the other only once.

Without loss of generality, let $c\left(w_{1}\right)=c\left(w_{2}\right)=2$ and $c\left(w_{3}\right)=3$. Now, $N_{2}\left(w_{1}\right)=$ $\left\{u, v_{2}, v_{3}\right\}, N_{2}\left(w_{2}\right)=\left\{u, v_{1}, v_{3}\right\} \& N_{2}\left(w_{3}\right)=\left\{u, v_{1}, v_{2}\right\}$. Hence, $c\left(v_{1}\right), c\left(v_{2}\right) \neq 2,3$ and therefore, $c\left(v_{1}\right)=c\left(v_{2}\right)=1$. Then all the three neighbors of $w_{3}$ are of color 1 and hence, $w_{3}$ cannot be an $e 2-c d v$. Since, $w_{3}$ is the only vertex of color 3, there does not exist a 3-e2-cdv. Hence, $H_{4}$ cannot have a $3-e 2 b$-coloring. Then $S_{e 2 b}\left(H_{4}\right)=\{2,4\}$ and $H_{4}$ is not $e 2 b$ continuous.

(ii) For $n \geq 5$, the $e 2 b$-continuity is discussed in three cases.

\section{Case (i): $5 \leq n \leq 7$}

If $n=5$, then $\chi_{e 2}\left(H_{n}\right)=3$ and $\chi_{e 2 b}\left(H_{n}\right)=n-2=3$. Hence, $\chi_{e 2 b}\left(H_{n}\right)=\chi_{e 2}\left(H_{n}\right)$ and $S_{e 2 b}\left(H_{n}\right)=\{3\}$. If $n=6$, then $\chi_{e 2}\left(H_{n}\right)=4$ and $\chi_{e 2 b}\left(H_{n}\right)=n-2=4$. Hence, $\chi_{e 2 b}\left(H_{n}\right)=$ $\chi_{e 2}\left(H_{n}\right)$ and $S_{e 2 b}\left(H_{n}\right)=\{4\}$. If $n=7$, then $\chi_{e 2}\left(H_{n}\right)=4$ and $\chi_{e 2 b}\left(H_{n}\right)=n-2=5$. Hence, $\chi_{e 2 b}\left(H_{n}\right)=\chi_{e 2}\left(H_{n}\right)+1$ and $S_{e 2 b}\left(H_{n}\right)=\{4,5\}$. It is clear that, $\chi_{e 2 b}\left(H_{n}\right)$ is either $\chi_{e 2}\left(H_{n}\right)$ or $\chi_{e 2}\left(H_{n}\right)+1$. Hence, $H_{n}$ is $e 2 b$-continuous.

Now suppose $n \geq 8$. The $e 2 b$-continuity of $H_{n}$ is checked for each $k, \chi_{e 2}\left(H_{n}\right)=\left\lfloor\frac{n}{2}\right\rfloor \leq k \leq$ $n-2=\chi_{e 2 b}\left(H_{n}\right)$. Hence, it is enough to verify the $e 2 b$-coloring with $k$ colors only for the intermediate values. i.e., for $k,\left\lfloor\frac{n}{2}\right\rfloor<k<n-2$, i.e., for $k,\left\lfloor\frac{n}{2}\right\rfloor+1 \leq k \leq n-3$. We show all the $e 2 b$-coloring of $H_{n}$ for the intermediate values, if there are atmost 2 intermediate values. The general proof is given for higher values. Now consider the value of $n$ satisfying $(n-2)-$ $\left\lfloor\frac{n}{2}\right\rfloor \leq 3$. Suppose $n$ is even. Then

$$
\begin{aligned}
(n-2)-\left\lfloor\frac{n}{2}\right\rfloor & \leq 3 \Leftrightarrow n-2-\frac{n}{2} \leq 3 \\
\Leftrightarrow & n \leq 10
\end{aligned}
$$

Hence, for $H_{8}$ and $H_{10}$, there are at most 2 intermediate values between $\chi_{e 2}\left(H_{n}\right)$ and $\chi_{e 2 b}\left(H_{n}\right)$.

Suppose $n$ is odd. Then $n-2-\left\lfloor\frac{n}{2}\right\rfloor \leq 3 \Leftrightarrow n-2-\left(\frac{n-1}{2}\right) \leq 3$

$$
\begin{gathered}
\Leftrightarrow \frac{n-3}{2} \leq 3 \\
\Leftrightarrow n \leq 9
\end{gathered}
$$

Since $n \geq 8, H_{9}$ has atmost 2 intermediate values between $\chi_{e 2}\left(H_{9}\right)$ and $\chi_{e 2 b}\left(H_{9}\right)$. Hence case (ii) follows.

Case (ii): $8 \leq n \leq 10$

If $n=8$, then $\chi_{e 2}\left(H_{n}\right)=\left\lfloor\frac{n}{2}\right\rfloor=4, \chi_{e 2 b}\left(H_{n}\right)=n-2=6$ and hence, the intermediate value is 5 . From figure $4, H_{8}$ has a 5 - $e 2 b$-coloring.

If $n=9$, then $\chi_{e 2}\left(H_{n}\right)=\left\lfloor\frac{n}{2}\right\rfloor=4, \chi_{e 2 b}\left(H_{n}\right)=n-2=7$ and hence, the intermediate values 


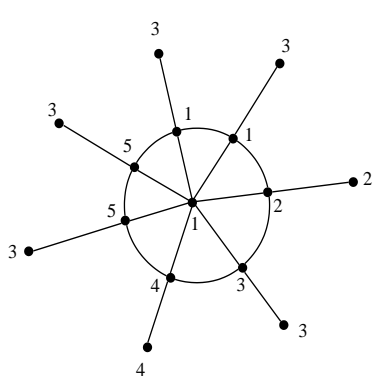

Figure 4: 5-e2b-coloring of $H_{8}$

are 5 and 6 . From figure $5, H_{9}$ has a 5 -e2b-coloring and from figure $6, H_{9}$ has a 6 -e2b-coloring. If $n=10$, then $\chi_{e 2}\left(H_{n}\right)=\left\lfloor\frac{n}{2}\right\rfloor=5, \chi_{e 2 b}\left(H_{n}\right)=n-2=8$ and hence, the intermediate values are 6 and 7 . From figures 7 and $8, H_{10}$ has a 6 -e2b-coloring and a 7-e2b-coloring.

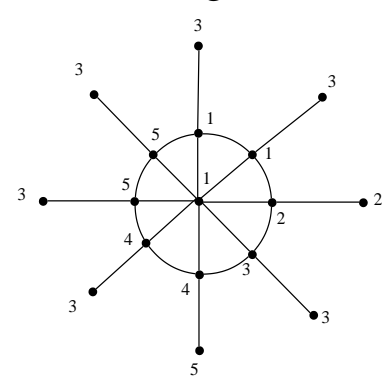

Figure 5: 5-e2b-coloring of $H_{9}$

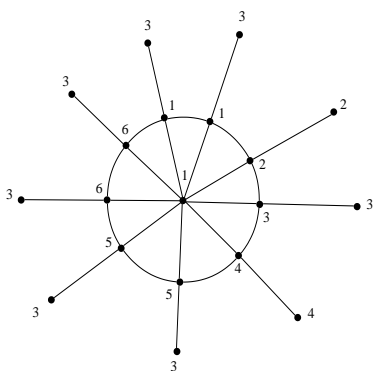

Figure 7: 6-e2b-coloring of $H_{10}$

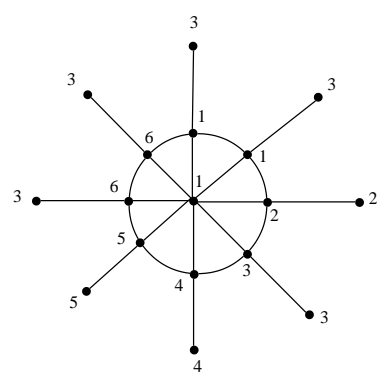

Figure 6: 6-e2b-coloring of $H_{9}$

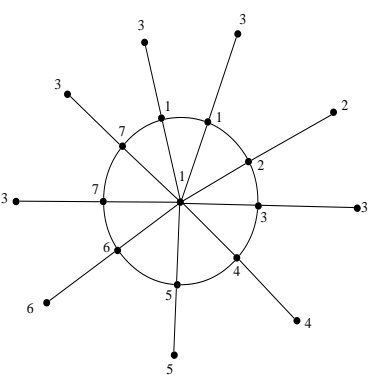

Figure 8:7-e2b-coloring of $H_{10}$

Case (ii): $n \geq 11$

In the $(n-2)-e 2 b$-coloring of $H_{n}$ on the $(n-1)$-cycle, $v_{1}$ and $v_{n-1}$ are of color 1 and the remaining $n-3$ vertices from $v_{2}$ to $v_{n-2}$ are of distinct colors. Hence, in an $e 2 b$-coloring of $H_{n}$ with $k$ colors, $\left\lfloor\frac{n}{2}\right\rfloor+1 \leq k \leq n-3$, on the $(n-1)$-cycle, leaving $v_{1}$ and $v_{n-1}$, the remaining $n-3$ vertices from $v_{2}$ to $v_{n-2}$ receive $k-1$ colors. Then

number of colors repeated $=(n-3)-(k-1)=n-k-2$

When a color is repeated, it occurs in two adjacent vertices. We combine pairs of vertices in the sequences $v_{2}, v_{3}, \ldots, v_{n-2}$ with higher suffices. Let vertices $v_{i}$ to $v_{n-2}$ be combined in a $k$ coloring. Clearly $v_{i}$ is the vertex of smallest index to receive the same color. Hence, it is necessary to find $v_{i}$ in each coloring in the specified interval. If $k$ colors are given, then from (4), $n-k-2$ colors are repeated. In other words, $2(n-k-2)$ vertices are combined pairwise. Hence, $i$ of $v_{i}$ is computed as follows

$i=(n-2)-[2(n-k-2)-1]=2 k-n+3$ 
Now to compute $v_{i}$ for the maximum and minimum values of $k$. When $k$ is maximum, $k=$ $n-3$, and from (5), $i=n-3$ and hence $v_{i}=v_{n-3}$. Suppose $k$ is minimum. Then $k=\left\lfloor\frac{n}{2}\right\rfloor+$ 1 .

$\therefore\left\lfloor\frac{n}{2}\right\rfloor+1 \leq k \leq n-3$

Hence, there are 2 values for $i$ depending on $n$ whether it is even or odd.

If $n$ is even, then $k=\frac{n}{2}+1$ and $i=2\left(\frac{n}{2}+1\right)-n+3=5$. Hence, vertices are combined from $v_{5}$ onwards.

If $n$ is odd, then $k=\frac{n-1}{2}+1$ and $i=2\left(\frac{n-1}{2}+1\right)-n+3=4$. Hence, vertices are combined from $v_{4}$ onwards.

In general, from (5), in a $k$-coloring of $H_{n}, v_{2}$ to $v_{2 k-n+2}$ are of distinct colors and vertices $v_{2 k-n+3}$ to $v_{n-2}$ are combined pairwise and given colors from $2 k-n+3$ to $k$. Now let $c: V \rightarrow\{1,2, \ldots, k\},\left\lfloor\frac{n}{2}\right\rfloor+1 \leq k \leq n-3$. First assign colors to $u, v_{1}$ and $v_{n-1}$ as in (7).

$c(u)=c\left(v_{1}\right)=c\left(v_{n-1}\right)=1$

In a $k$-coloring, leaving color $1, k-1$ colors are available for the $n-3$ vertices from $v_{2}$ to $v_{n-2}$. From (5), vertices from $v_{2 k-n+3}$ to $v_{n-2}$ are combined pairwise. Hence, $v_{2}$ to $v_{2 k-n+2}$ receive distinct colors. Let

$c\left(v_{i}\right)=c\left(w_{i}\right)=i, 2 \leq i \leq 2 k-n+2$

From (8), colors used from $v_{2}$ to $v_{2 k-n+2}=2 k-n+1$

$\therefore$ Colors available for $v_{2 k-n+3}$ to $v_{n-2}=k-1-(2 k-n+1)=n-k-2$

(9)

Number of vertices from $v_{2 k-n+3}$ to $v_{n-2}=n-2-(2 k-n+2)=2 n-2 k-4$

$\therefore$ Number of pairs from $v_{2 k-n+3}$ to $v_{n-2}=n-k-2$

Hence, from (9) \& (10), the $2 n-2 k-4$ vertices from $v_{2 k-n+3}$ to $v_{n-2}$ can receive $n-k-2$ colors from $2 k-n+3$ to $k-1$ pairwise. Now to color $v_{i}, 2 k-n+3 \leq i \leq n-2$. For $1 \leq$ $i \leq n-k-2,2 k-n+3 \leq 2 k-n+2 i+1 \leq n-3 \quad$ and $\quad 2 k-n+4 \leq 2 k-n+2 i+$ $2 \leq n-2$. Hence,

(i) if $n$ is even, then $2 k-n+2 i+1$ takes all odd values and $2 k-n+2 i+2$ even values between $2 k-n+3 \& n-2$ and

(ii) if $n$ is odd, then $2 k-n+2 i+1$ takes all even values and $2 k-n+2 i+2$ odd values between $2 k-n+3 \& n-2$.

Hence, from (i) and (ii), $2 k-n+2 i+1$ and $2 k-n+2 i+2$ assume all the values between $2 k-n+3 \& n-2$. Hence, the following fact.

Fact 1: Coloring $v_{i}, 2 k-n+3 \leq i \leq n-2$ is same as coloring $v_{2 k-n+2 i+1} \& v_{2 k-n+2 i+2}$, $1 \leq i \leq n-k-2$.

Now color $v_{i}^{\prime} s$ from $v_{2 k-n+3}$ to $v_{n-2}$, pairwise as described below.

$$
c\left(v_{2 k-n+2 i+1}\right)=c\left(v_{2 k-n+2 i+2}\right)=2 k-n+1+i, \quad 1 \leq i \leq n-k-2
$$

i.e., $c\left(v_{2 k-n+2 i+1}\right)=c\left(v_{2 k-n+2 i+2}\right)=2 k-n+i+1,1 \leq i \leq n-k-2$

Thus, all the vertices of $H_{n}$ but $w_{i}{ }^{\prime} s$ from $w_{2 k-n+3}$ to $w_{n-2}$ are colored and they are colored based on whether $n$ is even or odd. Before assigning colors to $w_{i}{ }^{\prime} s, c$ defined in (7), (8) \& (11) is collectively given in (12) 


$$
\left.\begin{array}{c}
c(u)=c\left(v_{1}\right)=c\left(v_{n-1}\right)=1 ; \\
c\left(v_{i}\right)=c\left(w_{i}\right)=i, 2 \leq i \leq 2 k-n+2 ; \\
c\left(v_{2 k-n+2 i+1}\right)=c\left(v_{2 k-n+2 i+2}\right)=2 k-n+i+2, \quad 1 \leq i \leq n-k-2 ; \\
c\left(w_{1}\right)=c\left(w_{n-1}\right)=3 .
\end{array}\right\}
$$

The remaining $w_{i}^{\prime} s, 2 k-n+3 \leq i \leq n-2$ are assigned colors as described below.

(i) If $n$ is even, let $c\left(w_{i}\right)=3,2 k-n+3 \leq i \leq n-2$.

(ii) If $n$ is odd, then for $\left\lfloor\frac{n}{2}\right\rfloor+2 \leq k \leq n-3$ and $2 k-n+3 \leq i \leq n-2$, let $c\left(w_{i}\right)=3$.

If $k=\left\lfloor\frac{n}{2}\right\rfloor+1$, then let $c\left(w_{i}\right)=3$, for $5 \leq i \leq n-2$ and $c\left(w_{4}\right)=5$.

Since, $v_{2}$ to $v_{2 k-n+2}$ receive colors from 2 to $2 k-n+2, v_{2 k-n+2}$ to $v_{n-2}$ receive colors from $2 k-n+3$ to $k$. Hence,

$2 k-n+3 \leq i \leq n-2 \Rightarrow 2 k-n+3 \leq c\left(v_{i}\right) \leq k$

Now to verify $c\left(v_{i}\right) \neq c\left(w_{i-1}\right), c\left(w_{i+1}\right)$.

Suppose $n$ is even. Then from (6), $k \geq \frac{n}{2}+1$. Hence,

$2 k-n+3 \geq 4$

If $i=1$, then $c\left(v_{i}\right)=1, c\left(w_{i-1}\right)=c\left(w_{n-1}\right)=3 \& c\left(w_{i+1}\right)=c\left(w_{2}\right)=2$.

If $i=2$, then $c\left(v_{i}\right)=2, c\left(w_{i-1}\right)=c\left(w_{1}\right)=3 \& c\left(w_{i+1}\right)=c\left(w_{3}\right)=3$.

If $3 \leq i \leq 2 k-n+1$, then $2 \leq i-1 \leq 2 k-n$ and $4 \leq i+1 \leq 2 k-n+2$. Hence,

$$
c\left(v_{i}\right)=i, c\left(w_{i-1}\right)=i-1 \& c\left(w_{i+1}\right)=i+1 .
$$

If $i=2 k-n+2$, then $c\left(v_{i}\right)=i=2 k-n+2, c\left(w_{i-1}\right)=c\left(w_{2 k-n+1}\right)=2 k-n+1$

and $c\left(w_{i+1}\right)=c\left(w_{2 k-n+3}\right)=3$

If $i=2 k-n+3$, then $c\left(v_{i}\right)=c\left(v_{2 k-n+3}\right)=c\left(v_{2 k-n+2 \times 1+1}\right)=2 k-n+1+2=2 k-$ $n+3$ and $c\left(w_{i-1}\right)=c\left(w_{2 k-n+2}\right)=2 k-n+2 \& c\left(w_{i+1}\right)=c\left(w_{2 k-n+4}\right)=3$.

If $2 k-n+4 \leq i \leq n-3$, then from (13) \& (14), $c\left(v_{i}\right) \geq 4$ and from (i), $c\left(w_{i-1}\right)=$ $c\left(w_{i+1}\right)=3$.

If $i=n-2$, then $c\left(v_{i}\right)=k \geq 4, c\left(w_{i-1}\right)=c\left(w_{n-3}\right)=3$ and $c\left(w_{i+1}\right)=c\left(w_{n-1}\right)=3$.

If $i=n-1$, then $c\left(v_{i}\right)=1, c\left(w_{i-1}\right)=c\left(w_{n-2}\right)=3$ and $c\left(w_{i+1}\right)=c\left(w_{1}\right)=3$.

Hence, $c\left(v_{i}\right) \neq c\left(w_{i-1}\right), c\left(w_{i+1}\right)$.

By similar discussion, $c\left(v_{i}\right) \neq c\left(w_{i-1}\right), c\left(w_{i+1}\right)$ can be proved if $n$ is odd. Thus the $k$ coloring $c$ defined is a 2-distance coloring. Since, for distinct colors $c\left(v_{i}\right)=c\left(w_{i}\right)$ and for pairwise colors $c\left(v_{i}\right)=c\left(v_{i+1}\right)$, each $v_{i}$ is an $e 2$-cdv. Hence, $H_{n}$ has an $e 2 b$-coloring for each $k$ between $\left\lfloor\frac{n}{2}\right\rfloor$ to $n-2$ and hence, $H_{n}$ is $e 2 b$-continuous.

\section{Conclusion}

In this paper, we introduced exact 2-distance $b$-continuous graph and exact 2-distance $b$-spectrum. Also, the exact 2-distance $b$-chromatic number of Helm graph $H_{n}$ was obtained. Further it is proved that for $n=4, H_{n}$ is not $e 2 b$-continuous and for $n \geq 5, H_{n}$ is $e 2 b$ continuous and there by proving there existe $2 b$-continuous graphs and graphs which are not $e 2 b$-continuous. 


\section{REFERENCES}

[1] F.Harary, Graph Thoery, Narosa /Addison Wesley, Indian Student Edition, 1988.

[2] R.W. Irving and D.F. Manlove, The b-chromatic number of a graph, Discrete. Appl. Math.

91 pp. 127-141, 1991.

[3] T. N. Janakiraman, M.Poobalaranjani and A. SenthilThilak, Exact k-distance colouring of

Graphs, Proceedings of the UGC sponsored National Seminar on Applications in Graph Theory

[4] F. Kramer and H. Kramer, Un probl'eme de coloration des sommets d'un graphe. C. $R$. Acad. Sci. Paris A 268(7), pp. 46-48, 1969.

[5] F. Kramer and H. Kramer, EinFarbungsproblem der KnotenpunkteeinesGraghenbezuglich der Distanz p, Rev. Roumaine Math. PuresAppln., 14(2), pp.1031-1038, 1969.

[6] Nesetril J, Ossona de Mendez, Sparsity, Graphs, Structures, and Algorithms, Springer Verlag, Berlin, Heidelberg, 2012.

[7] S. Saraswathi and M.Poobalaranjani, Exact 2-distance b-coloring of some classes of graphs,

Malaya Journal of Matematik, Vol. 8, No. 1, 195-200, 2020 MJM 08010033 